Proceedings

\title{
Tools for Planning Energy Efficient District Systems ${ }^{\dagger}$
}

\author{
Miika Rämä * and Krzysztof Klobut \\ VTT Technical Research Centre of Finland Ltd., Vuorimiehentie 3, 02150 Espoo, Finland; \\ krzysztof.klobut@vtt.fi \\ * Correspondence: miika.rama@vtt.fi; Tel.: +358-40-592-4000 \\ + Presented at Sustainable Places 2018 (SP 2018), Aix-les Bains, France, 27-29 June 2018. \\ Published: 23 August 2018
}

\begin{abstract}
An open-source planning tool for the evaluation district cooling systems is developed within project INDIGO. The tool is based on optimisation model consisting of a defined set of components in a district cooling (DC) system. The approach links up the whole energy chain from consumption to resources achieve an optimal solution. The tool will enable assessment on effects of single components on a system level and provide data for comparison from energy efficiency, economic feasibility and the climate impact point of view. Life cycle assessment (LCA) framework will be utilised as a method for both economic feasibility and climate impact evaluation. This paper reviews the related projects and positions INDIGO planning tool in this context.
\end{abstract}

Keywords: district cooling; energy planning; energy systems

\section{Introduction}

The goal of project INDIGO is to provide tools supporting design, planning and operation of DC systems. The project covers all the parts of the system; generation, distribution, storage, and demand [1]. The focus of this paper is on the planning of a DC or a district heating (DH) system. It presents the other ongoing projects of a similar topic, describes in more detail the planning tool being developed within INDIGO and how they all contribute to the state of the art.

\section{Review of Related Research}

The OPTi project [2] will deliver methodologies and tools that will enable accurate modelling, analysis and control of current and envisioned district heating and cooling (DHC) systems. The methodology will be deployed both on a complete system level, and on the level of a building(s). The DHC system will be a subject to dynamic control, and will treat thermal energy as a resource to be controlled for saving energy and reducing peak load. OPTi-Sim facilitates a virtual representation of the real DHC system and is a central component of the OPTi framework. It integrates different models in a co-simulation approach.

FLEXYNETS project [3] will develop, demonstrate and deploy a new generation of intelligent DHC network that reduces energy transportation losses by using low distribution temperature levels $\left(15-20^{\circ} \mathrm{C}\right)$. The network is used as a source of both heating and cooling by utilising heat pump technology. GIS Software Tool for Designing Networks is being developed. The analysis is based on steady state conditions for fixed temperature levels. 
In STORM project [4] a generic DHC network controller will be developed with the ambition to increase the use of excess heat and renewable energy sources in the DHC system. The self-learning control is used instead of model-based control approaches. It will make the controller easy to implement in different configuration and generations of DHC networks. The STORM controller includes modules for forecasting, planning and dispatching heating and cooling production. The control strategies benefit the whole energy chain, from generation and distribution to consumption.

InDeal project [5] will offer an innovative platform that will impose an adequate distribution of heating and cooling among the buildings in the network. A number of modules have been integrated in an advanced automated Decision Support System (DSS) including artificial intelligence metering devices, energy demand prediction of the network, data-driven short-term weather forecasting, energy storage monitoring and modelling, energy harvesting, web-based control platform along with solutions for minimizing heat losses.

The innovative thermo-chemical network technology of H-DisNet project [6] will contribute to the next-generation district energy networks. The technology will exploit high chemical potential of absorption processes for loss-free transport and storage of energy potential. The concept forms an intelligent district network with thermal, electric and gas networks. The modelling of H-DisNet will provide the input for control strategies, large network examination and potential assessment.

E2District project [7] aims to develop and demonstrate a novel cloud enabled management framework for DHC systems, which will deliver energy cost savings through intelligent adaptive DHC control and optimisation methods. A scalable District Operation System (DOS) will integrate all control, optimisation, diagnostics and prosumer engagement into a DHC management platform.

The objective of CoolHeating [8] is to support the implementation of "small modular renewable heating and cooling grids" for communities in South-Eastern Europe. Economic calculation tool has been developed for small modular district heating and cooling projects, mainly meant for non-experts of DH technology, e.g., decision-makers in municipalities. Tool focuses on economic aspects of the system and the main input consists of investment and financing, operational costs, revenues and economic parameters. The results include assets, liabilities and equities, incomes, a balance sheet, cash flow and profitability.

PLANHEAT project [9] aims to develop a tool for mapping, planning and simulating a local energy demand and supply to investigate future scenarios, potential identification of an existing network and describe benefits from energetic, economic and environmental points of view. Tool will be easy-to-use, open-source and free. Potential mapping includes demand estimation (degree hours for cooling and heating), database for retrofitting and energy efficiency measures, models for current and future energy demand estimation, available energy sources and industrial excess heating and cooling. GIS based platform will be developed to visualize potential of both demand and supply.

SDHp2m project [10] mission is to develop, improve and implement advanced policies and support measures for Solar District Heating (SDH) in 9 participating European regions. On their website, evaluations and a number of calculation tools dedicated to solar district heating can be found enabling feasibility studies. For example, the SDH Online Calculation Tool is based on TRNSYS simulation results and provides a first idea of dimensioning and economics of a solar district heating plant. The tool is suitable for centralised distributed solar district heating plants.

The aim of THERMOSS [11] is to define a set of retrofitting heating and cooling packages based on cutting-edge, high-potential, market-ready technologies that are connected together in an open ICT platform for smart energy management at building and district level. It employs DIMOSIM, an integrated simulation tool for the analysis of feasibility, conception and operation of district energy systems. The tool is meant for the development, test and analysis of the optimisation of concepts and operation of district energy systems.

The THERMOS project [12] aims to provide the methods, data, and tools (free and open source) to enable more sophisticated thermal energy system planning rapidly and cheaply. The THERMOS Tool is a map-driven open-source web-based application tailored to the real world requirements of energy planners to make heat network planning faster and more efficient. 
HotMaps project [13] will produce a web-based GIS toolbox for analyzing heating and cooling demand as well as supply options on a local, regional and national levels. All the source code produced during the development of Hotmaps will be accessible on the open source platform GitHub. Main features will be to identify location of heating and cooling demand and potential supply on a map (EU28) with renewable and industrial excess potential highlighted, to estimate potential district heating options for a selected area, to estimate and compare costs of individual heating vs. district heating and to compare the results with national plans for de-carbonisation.

District Heating Assessment Tool (DHAT) [14] is a tool for economic feasibility studies for establishing district heating with a comparison with individual heating. It can be adjusted to local conditions globally. The model can be used to show the economic and environmental benefits of $\mathrm{DH}$. The tool is based on Danish technical data and price projections which can be adjusted, making it applicable to other countries as well. The model is based on MS Excel with no hidden data or VBA code - the user is free to modify all data inputs and variables.

\section{Introduction to INDIGO Planning Tool}

The INDIGO planning tool will provide means for evaluating the performance, benefits and potential of DC systems. The aim is to create an easy-to-use open-source tool that enables analysis of a cooling system in defined area with a group of buildings connected and a comparison with a building or space specific cooling solution. Based on the background review in Section 2 concerning the tools developed in other projects in, the planning tool complements well the other solutions.

The tool generates an energy system model based on user input. The components defined can be categorised into five groups; resources, energy supply, cooling production, distribution and consumption, illustrated in Figure 1. This optimization model is then solved for the energy consumption of a representative full year of operation. The results are further used for a Life Cycle Assessment (LCA) to obtain both economic and climate impact analysis for the system [15].

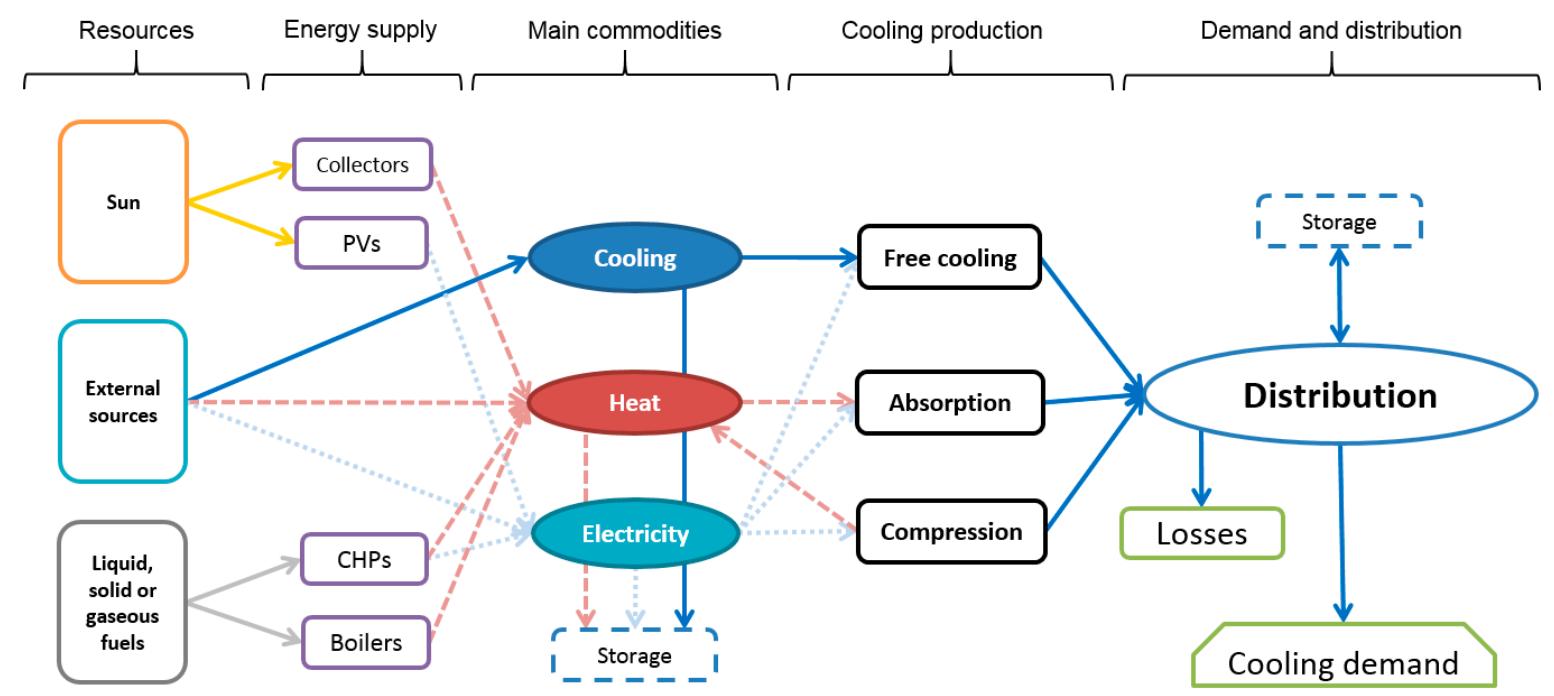

Figure 1. Structure and elements of the energy system model representing a cooling system.

The analysis is divided into three parts; energy, economic and environmental analysis.

Energy analysis finds out how the specified system is operated and what is its primary energy consumption. The results is a product of an optimisation model generated using the information on system definition, and thus the energy analysis is actually a techno-economic analysis as prices of the commodities used (e.g., electricity) is taken into account. The modelling library used for the optimisation model is Oemof ("Open Energy System Modelling Framework") [16].

In economic analysis, the related investment costs are taken into account and the profitability or accumulated costs of the system are calculated over a specified life time. 
The environmental analysis in the planning tool focuses on climate impacts of the district cooling systems in comparison to alternative cooling systems. The climate impact assessment will follow the framework of LCA. Thus, the LCA will provide information on potential emission savings achieved with the district cooling systems. In addition, it helps the user to recognise the most emission intensive components and functions of the system being studied, and thus enable efficient emission reduction measures. As LCA concentrates on all the inflows and outflows of substances it also reduces the risk of problem shifting, i.e., situations where an improvement in one part of the life cycle leads to weakening in another part.

Combining the results of the different analysis phases, combination indicators such as price of cooling or comparative indicators such as costs of emission reduction can be calculated. The planning tool will also include functionalities for performing a sensitivity analysis.

Program implementation in Python will take advantage of object-oriented programming for data structures and storage. The predefined input data will be stored in csv files.

Graphic user interface (GUI) of the planning tool is used to manage model data, structure, and pass these values into optimization module of the tool, and import result data from optimization in order to form easy to read result summary. GUI consists of five functional tabs: Analysis, GIS Tool, Parameters, Results and Sensitivity Analysis. GIS tool of GUI is utilised in forming simple but functional network of cooling system based on map file of the area analysed.

\section{Conclusions}

There is a number of projects developing tools for assessment of DHC systems. The tool being developed within INDIGO is comparable the products of related projects. Although focusing only on DC and cooling systems in general, the approach and capabilities seem comprehensive, analyzing the system from all energy, economic and environmental perspectives. The later phases of the INDIGO project include task for applying the tool for a selected group of case systems, validating the usefulness of the concept.

Currently available reports of the tool describe (1) the system specification setting the boundaries and scope for the analysis; (2) the methods used for energy, economic and environmental analysis; (3) the input data requirements set by the system definition and the implementation plans.

Funding: This research received no external funding other than that mentioned in Acknowledgements section below.

Acknowledgments: Project INDIGO has received funding from European Union's Horizon 2020 research and innovation programme under grant agreement $n^{\circ} 69698$.

Conflicts of Interest: The authors declare no conflict of interest.

\section{References}

1. Passerini, F.; Sterling, R.; Keane, M.; Klobut, K.; Costa, A. Energy efficiency facets: Innovative district cooling systems. Entrep. Sustain. Issues 2017, 4, 310-318, doi:10.9770/jesi.2017.4.3S(6).

2. OPTi Project. Available online: http://www.opti2020.eu (accessed on 5 July 2018).

3. FLEXYNETS Project. Available online: http://www.flexynets.eu (accessed on 5 July 2018).

4. STORM Project. Available online: http://www.storm-dhc.eu (accessed on 5 July 2018).

5. InDeal Project. Available online: http://www.indeal-project.eu (accessed on 5 July 2018).

6. H-DisNet Project. Available online: http://www.h-disnet.eu (accessed on 5 July 2018).

7. E2District Project. Available online: http://e2district.eu (accessed on 5 July 2018).

8. CoolHeating Project. Available online: http://www.coolheating.eu (accessed on 5 July 2018).

9. PlanHeat Project. Available online: http://planheat.eu (accessed on 5 July 2018).

10. SDHp2m Project. Available online: http://solar-district-heating.eu (accessed on 5 July 2018).

11. THERMOSS Project. Available online: http://thermoss.eu (accessed on 5 July 2018).

12. THERMOS Project. Available online: http://www.thermos-project.eu (accessed on 5 July 2018).

13. HotMaps Project. Available online: http://www.hotmaps-project.eu (accessed on 5 July 2018). 
14. DHAT Tool. Available online: https://ens.dk/en/our-responsibilities/global-cooperation/district-heatingassessment-tool-dhat (accessed on 5 July 2018).

15. Rämä, M.; Klobut, K.; Pursiheimo, E.; Koponen, K.; Sokka, L. District Cooling Planning Tool Framework and Specification; INDIGO project deliverable D5.1; 2017. Available online: http://www.indigoproject.eu/results/publicdeliverables/ (accessed on 15 August 2018).

16. Oemof Developers. Available online: http://oemof.readthedocs.io/en/stable/ (accessed on 17 July 2018).

(C) 2018 by the authors. Licensee MDPI, Basel, Switzerland. This article is an open access article distributed under the terms and conditions of the Creative Commons Attribution (CC BY) license (http://creativecommons.org/licenses/by/4.0/). 